\title{
Telomere Length Attrition, a Marker of Biological Senescence, Is Inversely Correlated with Triglycerides and Cholesterol in South Asian Males with Type 2 Diabetes Mellitus
}

\author{
Alison L. Harte, ${ }^{1}$ Nancy F. da Silva, ${ }^{1,2}$ Michelle A. Miller, ${ }^{1}$ Francesco P. Cappuccio, ${ }^{1}$ \\ Ann Kelly, ${ }^{3}$ Joseph P. O'Hare, ${ }^{1}$ Anthony H. Barnett, ${ }^{3}$ Nasser M. Al-Daghri, ${ }^{4,5}$ \\ Omar Al-Attas, ${ }^{4,5}$ Majed Alokail, ${ }^{4,5}$ Shaun Sabico, ${ }^{4}$ Gyanendra Tripathi, ${ }^{1}$ Srikanth Bellary, ${ }^{3,6}$ \\ Sudhesh Kumar, ${ }^{1}$ and Philip G. McTernan ${ }^{1}$ \\ ${ }^{1}$ Clinical Sciences Research Laboratories, Division of Metabolic and Vascular Health, Warwick Medical School, University of Warwick, \\ University Hospital Coventry and Warwickshire, Coventry CV2 2DX, UK \\ ${ }^{2}$ AstraZeneca R\&D, CVGI Bioscience, Mereside, Alderley Park, Macclesfield, Chesire SK1 04TG, UK \\ ${ }^{3}$ University of Birmingham and Biomedical Research Centre, Heart of England NHS Foundation Trust, Birmingham B4 7ET, UK \\ ${ }^{4}$ Biomarkers Research Program, Biochemistry Department, College of Science, King Saud University, Riyadh 11451, Saudi Arabia \\ ${ }^{5}$ Center of Excellence in Biotechnology, King Saud University, Riyadh 11451, Saudi Arabia \\ ${ }^{6}$ Aston University, Birmingham B4 7ET, UK
}

Correspondence should be addressed to Philip G. McTernan, p.g.mcternan@warwick.ac.uk

Received 17 September 2011; Revised 14 November 2011; Accepted 21 November 2011

Academic Editor: Toshiyasu Sasaoka

Copyright (c) 2012 Alison L. Harte et al. This is an open access article distributed under the Creative Commons Attribution License, which permits unrestricted use, distribution, and reproduction in any medium, provided the original work is properly cited.

South Asians have a higher risk of type 2 diabetes mellitus (T2DM) and cardiovascular disease (CVD) than white Caucasians, for a given BMI. Premature biological ageing, assessed by reduction in telomere length (TL), may be mediated by factors resulting from altered metabolic profiles associated with obesity. We hypothesise that ethnicity and metabolic status represent detrimental factors contributing to premature biological ageing. Therefore we assessed TL in two South Asian, age and BMI-matched cohorts [T2DM $(n=142)$ versus non-T2DM $(n=76)]$ to determine the effects of BMI, gender, lipid and CVD profile on biological ageing. Genomic DNA was obtained from the UKADS cohort; biochemical and anthropometric data was collected and TL was measured by quantitative real-time PCR. Our findings indicated a gender-specific effect with reduced TL in T2DM men compared with non-T2DM men $(P=0.006)$. Additionally, in T2DM men, TL was inversely correlated with triglycerides and total cholesterol $(r=-0.419, P<0.01 ; r=-0.443, P<0.01)$. In summary, TL was reduced amongst South Asian T2DM men and correlated with triglycerides and total cholesterol. This study highlights enhanced biological ageing among South Asian, T2DM men, which appears to be tracked by changes in lipids and BMI, suggesting that raised lipids and BMI may directly contribute to premature ageing.

\section{Introduction}

People of South Asian origin have a higher predisposition to the development of obesity-related diseases, including type 2 diabetes mellitus (T2DM) when compared with white Caucasians and other ethnicities [1,2]. Increased metabolic risk among South Asians has been linked to significantly higher leptin levels when compared to other ethnic groups and leptin is a predictor for cardiovascular risk and T2DM.
Heightened leptin levels in South Asians may be due to the higher body fat percentage, with more pronounced abdominal obesity compared with other ethnic groups matched for the same body mass index (BMI). This unfavourable body composition promotes decreased insulin sensitivity in this racial group [3]. Dyslipidaemia, specifically low high-density lipoprotein cholesterol (HDL-cholesterol), is also more common among South Asians compared with Europeans and East Asians [4]. These differences in metabolic phenotypes 
shed light on ethnic discrepancies in risk assessment and support the need for customized screening and diagnosis to optimize treatment. We have previously demonstrated that culturally tailored diabetes care among South Asians leads to enhanced health outcomes as compared to conventional standard care [5].

Telomere length (TL) may play an important role in understanding premature ageing due to the influences of metabolic parameters across ethnic groups. Telomeres are tandem repeats of DNA sequences (TTAGGG) at the end of chromosomes that play an important part in the biology of eukaryotic cells [6], a role as biomarkers of senescence and ageing has been established in many studies, with TL decreasing with advancing age [7-9]. Furthermore, ethnic differences in TL have been observed in different ethnic groups, with black Afro-Caribbean and Hispanic ethnic groups showing greater differences in age-associated TL than white Caucasians [10]. TL has also been associated with many modifiable factors such as lifestyle and marital status $[11,12]$. More importantly, there is a significant influence of age-related diseases such as coronary heart disease (CHD), T2DM and cancer on TL reduction [13-16].

Several studies confirm the presence of shorter TL among various ethnicities in patients with T2DM and insulin resistance $[15,17]$. To our knowledge, no study has reported data on South Asians with respect to TL and metabolic state.

Our cross-sectional study aims to determine whether premature biological ageing, assessed by reduction in TL, is evident in a cohort phenotypically predisposed to develop insulin resistance. Specifically, we aim to identify associations between TL and BMI, gender, lipid, and cardiovascular profile in both T2DM and non-T2DM South Asians.

\section{Materials and Methods}

2.1. Subjects. Age- and BMI-matched T2DM and subjects, aged between 45-60 years old, were randomly selected from the UK Asian Diabetes Study (UKADS, T2DM) [18, 19] and from the Wandsworth Heart and Stroke Study (WHSS, nonT2DM) $[20,21]$. The selection of the age range was utilized to ensure sufficient power for the study and based on the UK Prospective Diabetes Study (UKPDS) population matching the non-T2DM cohort. Biochemical and anthropometric data were collected for T2DM and non-T2DM subjects, which are detailed in tables 1 and 2. Routine T2DM management, dietary intervention, first line therapy (metformin and combination fibrates), as deemed clinically appropriate, were implemented.

2.2. UKADS and WHSS Plasma and Genomic DNA Extraction. Venous blood $(2 \times 9 \mathrm{~mL})$ per subject was collected in EDTA-coated vacutainers and allowed to stand upright at $4^{\circ} \mathrm{C}$ overnight. Plasma $(1 \mathrm{~mL})$ was removed from each tube and stored at $-80^{\circ} \mathrm{C}$ until required. The remaining blood samples were used for DNA extraction. Genomic DNA was extracted using an adaptation of the Nucleon BACC2 protocol (GE Healthcare, Little Chalfont, Buckinghamshire, UK). The DNA was resuspended in $200 \mu \mathrm{L}$ of TE buffer $(10 \mathrm{mM}$
Tris, $0.1 \mathrm{mM}$ EDTA, $\mathrm{pH}$ 8) and quantified by fluorometry using PicoGreen dye (Invitrogen-Molecular Probes, Paisley, UK) as well as by spectrophotometry, using the Nanodrop ND-1000 (NanoDrop Technologies, Wilmington, DE, USA).

2.3. TL Measurement. TL was measured by quantitative realtime polymerase chain reaction (RT-PCR) using the iQCycler real-time PCR system (Bio-Rad Laboratories, Hercules, CA, USA) [22]. In brief, TL was measured using the following telomere primers: primer Tellb (5' CGGTTTGTTTGGGTTTGGGTTTGGGTTTGGGTTTGGGTT $3^{\prime}, 450 \mathrm{nM}$ ), Tel2b (5' GGCTTGCCTACCCTTACCCTTACCCTTACCCTTACCCT 3', $450 \mathrm{nM}$ primer), $10 \mathrm{ng}$ genomic DNA, 2xiQ SYBR Green Supermix (Bio-Rad Laboratories, Hercules, CA, USA) in a $30 \mu \mathrm{L}$ reaction. The reactions were set up in quadruplicate in 96-well plates non-T2DM subjects. For each plate four DNA quantity standards (serial dilution ranging from 15 and $1.87 \mathrm{ng}$ per $30 \mu \mathrm{L}$ reaction) and three TL standards were also analysed. The three TL standards included $15 \mathrm{ng}$ of genomic DNA from isogenic human thyroid-derived cell lines: K1E72 (TL 3110 bp) and K1E71 (TL 3440 bp) and from a human lung fibroblast cell line MRC5 (TL $8470 \mathrm{bp}$ ). Standard curves were derived to convert the threshold cycle number $(\triangle \mathrm{CT})$ into starting quantity (SQ) of DNA and to convert the telomere/single copy gene SQ ratio to TL in base pairs (bp) [21-23]. The PCR cycle conditions were as follows: $95^{\circ} \mathrm{C}$ for $3 \mathrm{~min}, 40$ cycles of $95^{\circ} \mathrm{C}$ for $15 \mathrm{~s}$ and $56^{\circ} \mathrm{C}$ for $60 \mathrm{~s}$. In order to confirm the PCR specificity, a melting curve (dissociation curve) was performed by 46 repeats of increasing temperature, as previously described [22]. TL is expressed as mean \pm standard deviation $(\mathrm{SD})$.

2.4. Measurement of Insulin, Glucose, and Lipid Profile Levels. Plasma samples were analyzed for determination of insulin protein concentrations by a solid phase enzyme linked immunosorbant assay (Linco Research Inc. Missouri, USA; Insulin CV intraassay $5.96 \%$, interassay $10.3 \pm 0.9 \%$ ). Glucose concentration was determined by a glucose oxidase method (YSI 200 STAT Plus, YSI Incorporated, Yellow Springs, $\mathrm{OH}$, USA). Lipid profile was measured by the Clinical Biochemistry Laboratory at the University of Birmingham/ Heartlands/Queen Elizabeth Hospitals.

2.5. Statistical Analysis. Linear regressions, graphical functions, intergroup comparisons and Pearson's correlations were performed using the Statistical Package for the Social Sciences software (SPSS version 14.0 SPSS Inc., Chicago, IL, USA). TL and triglyceride data displayed a non-Gaussian distribution; therefore both data sets were accordingly log transformed prior to analysis. TL is represented by mean \pm $\mathrm{SD}$ and adjusted for age.

\section{Results}

$T L$ in subjects with T2DM. The general characteristics of both groups were presented in Tables 1 and 2. Mean TL for T2DM South Asian subjects was $5811.03 \pm 1945.52 \mathrm{bp}\left(\log ^{10}\right.$ $\mathrm{TL}: 3.74 \pm 0.13)$. We also examined TL for gender differences 
TABLE 1: General characteristics of the T2DM group (UKADS).

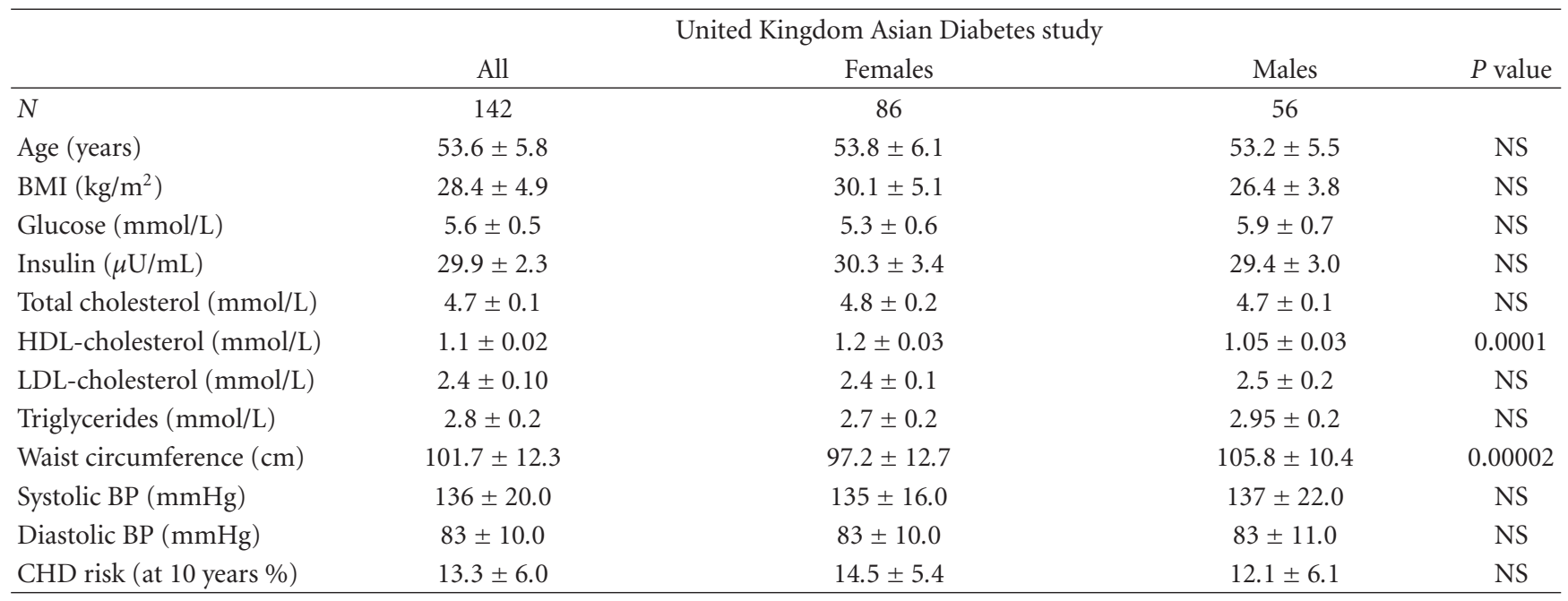

Note: NS: nonsignificant; CHD: cardiovascular heart disease.

TABLE 2: General characteristics of non-T2DM subjects (WHSS).

\begin{tabular}{lcccc}
\hline & & Wandsworth Heart and Stroke study & & \\
& All & Females & Males & \\
\hline$N$ & 76 & 33 & 43 & \\
Age $($ years $)$ & $49.81 \pm 5.23$ & $49.67 \pm 5.27$ & $49.93 \pm 5.26$ & $\mathrm{NS}$ \\
BMI $\left(\mathrm{kg} / \mathrm{m}^{2}\right)$ & $25.74 \pm 3.61$ & $27.53 \pm 3.90$ & $24.38 \pm 2.67$ & $\mathrm{NS}$ \\
\hline
\end{tabular}

Note: NS, nonsignificant.

and noted that males had a mean TL of $6040.64 \pm 2004,37 \mathrm{bp}$ $\left(\log ^{10}\right.$ TL: $\left.3.76 \pm 0.11\right)$ while females had $5608.61 \pm 1882.12 \mathrm{bp}$ $\left(\log ^{10} \mathrm{TL}: 3.73 \pm 0.14\right)$. TL did not display gender dimorphism in T2DM South Asian subjects $(P=\mathrm{NS})$. Age and BMI adjusted comparisons revealed that male T2DM subjects had significantly reduced TL compared with their non-T2DM counterparts $\left(P=0.008\left(\log ^{10} \mathrm{TL}\right)\right.$, Figure $\left.1(\mathrm{a})\right)$. This difference was not observed in the female cohort. Mean TL of T2DM was significantly reduced as compared with nonT2DM South Asians $\left(P=0.009\left(\log ^{10} \mathrm{TL}\right)\right.$, (Figure $\left.1(\mathrm{~b})\right)$.

3.1. Lipid Profile and TL in South Asian T2DM Subjects. Given that T2DM was associated with a reduction in TL in South Asians, further analysis investigated whether altered lipid profile had an effect on TL. Total cholesterol, LDLcholesterol, and triglycerides showed no gender differences (Table 1), but HDL-cholesterol was significantly higher in females compared to males $(P=0.0001$ Table 1$)$. There was no relationship overall between the lipid profiles for the T2DM group (Figures 2(a) and 3(a), data not shown, resp.), but when the cohort was stratified by gender, total cholesterol was inversely associated with TL in T2DM males, even after adjusting for age $(r=-0.414, P=0.001$; age adjusted: $r=-0.419, P=0.0018)$. Triglycerides showed a similar pattern, demonstrating an inverse association with age-adjusted TL of T2DM males $(r=-0.355, P=0.008$; age adjusted: $r=-0.386, P=0.007)$. These associations were not observed in the female group (Figures 2(b) and $3(\mathrm{~b}))$. Further subgroup analysis, according to BMI, in the male cohort highlighted that in obese subjects triglycerides inversely correlated with TL $(r=-0.336, P=0.038$; age adjusted: $r=-0.388, P=0.016 ; n=39$; Figure 4$)$. The rest of the associations were noncontributory with no significant effects noted by the use of medication.

\section{Discussion}

In our study, reduced telomere length (TL) observed in T2DM South Asian males was inversely associated with total cholesterol and triglycerides, even after adjusting for confounders. Shortened TL in T2DM subjects has previously been observed in Afro-Caribbeans [14], Arabs [15], and Caucasian subjects [17]. Information regarding TL and T2DM among South Asians is scarce, although in this population shortened TL has been observed for other age-related diseases, especially in vascular diseases (coronary artery disease) and hypertension, and cancer $[13,23,24]$. We observed that non-T2DM male subjects had, on average, shorter TL than non-T2DM females, confirming gender dimorphism which was also observed in other ethnic groups (Europeans, Japanese, as well as Arab children) [25-27]. The nonsignificant associations of TL to other parameters in this study, such as age, BMI and waist circumference, do not supersede 


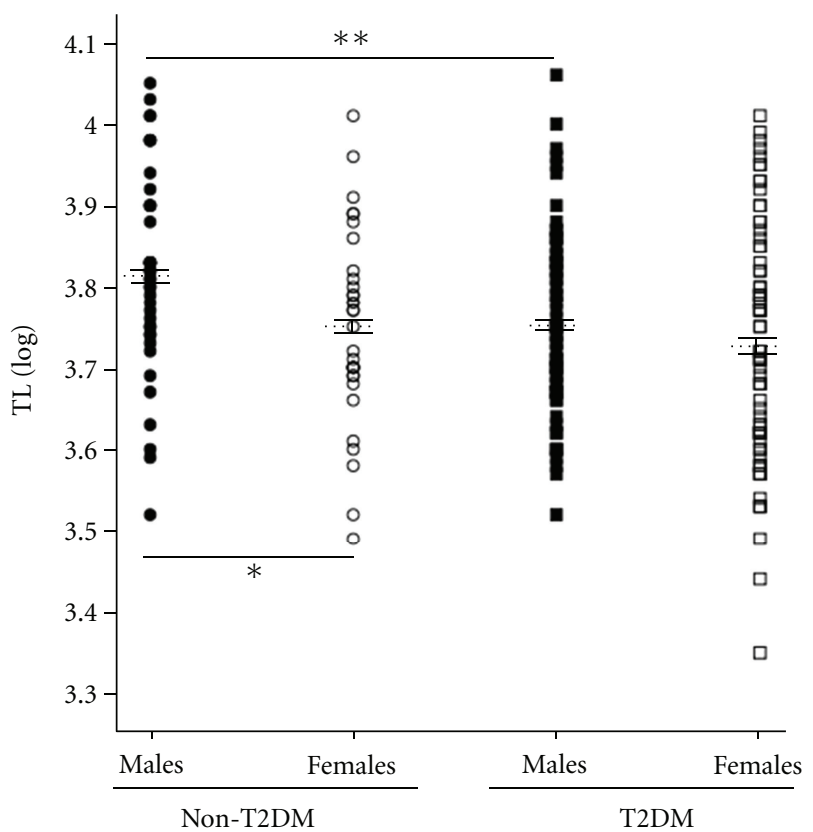

(a)

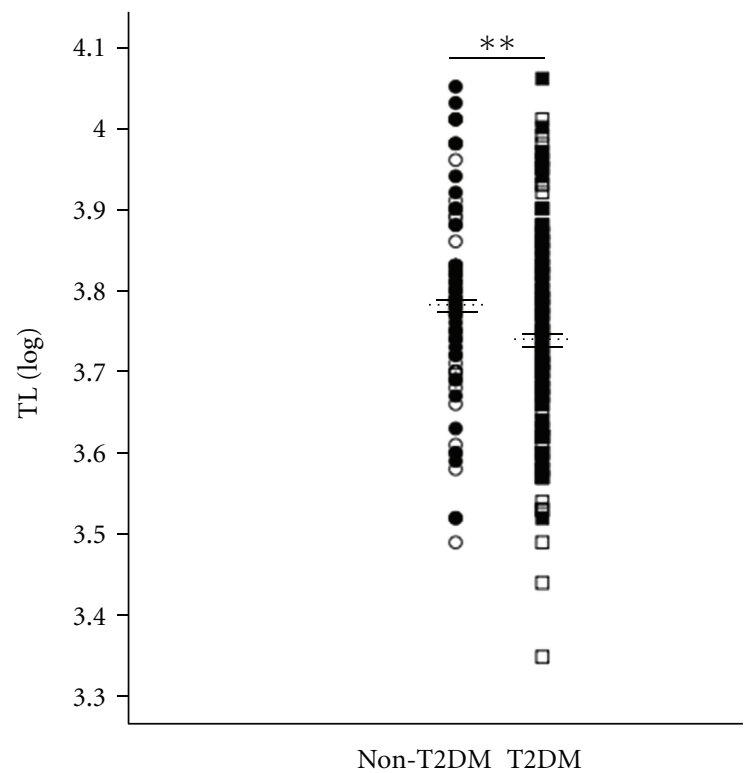

(b)

FIGURE 1: (a) Gender distribution of telomere length (TL) in peripheral blood mononuclear cells in South Asian non-T2DM (male: dark circles, females: open circles) and T2DM (male: dark squares, females: open squares); (b) TL in peripheral blood mononuclear cells in South Asian non-T2DM (circles) and T2DM (squares). TL is represented as mean (dotted line) $\pm \mathrm{SD},{ }^{*} P<0.05$ and ${ }^{* *} P<0.01$.

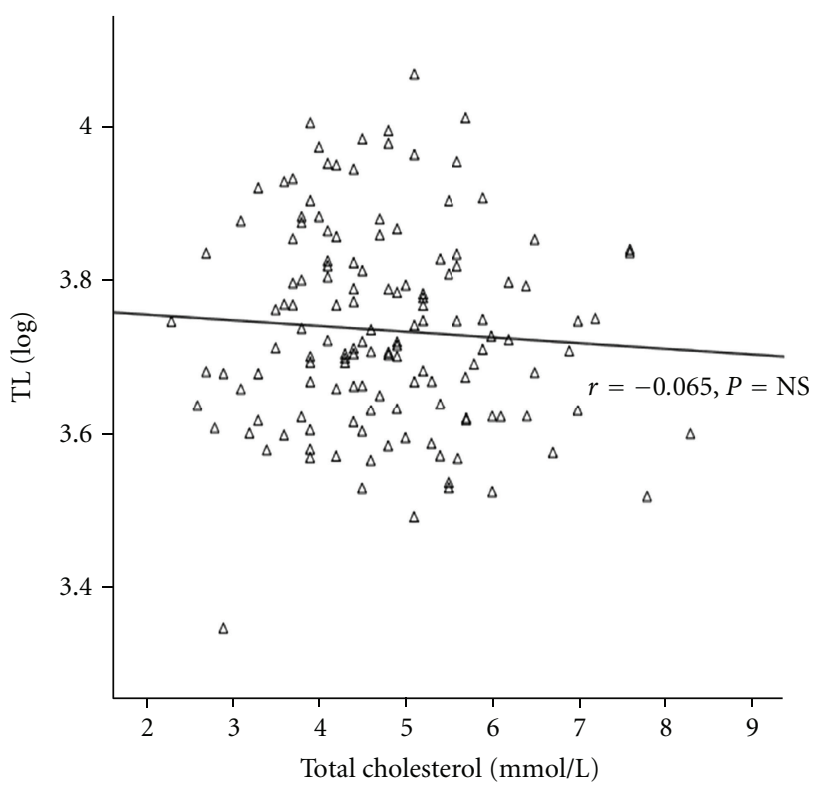

(a)

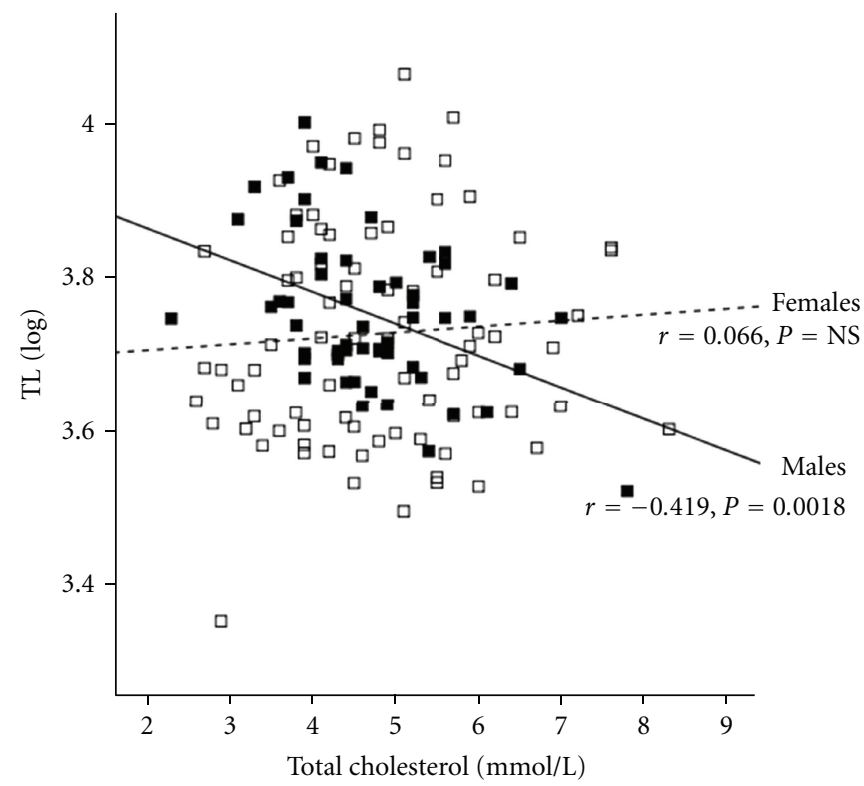

(b)

FIGURE 2: The relationship between total cholesterol and TL in South Asian T2DM group. (a) Linear regression analysis of total cholesterol and TL in T2DM (clear triangles); (b) linear regression analysis of total cholesterol and TL in T2DM by gender (male: dark squares and solid line, females: open squares and dotted line).

prior findings associating TL to age and obesity [15, 27, 28], since it is most likely that the present study's cohort did not reach enough power to elicit significance, or possibly due to the wide range of interindividual variation of TL [27].
The exact mechanism of TL attrition among patients with T2DM is largely explained by insulin resistance, a metabolic condition that promotes reactive oxygen species (ROS) formation and favours a proinflammatory milieu, two factors known to reduce lifespan [29]. One of the most important 


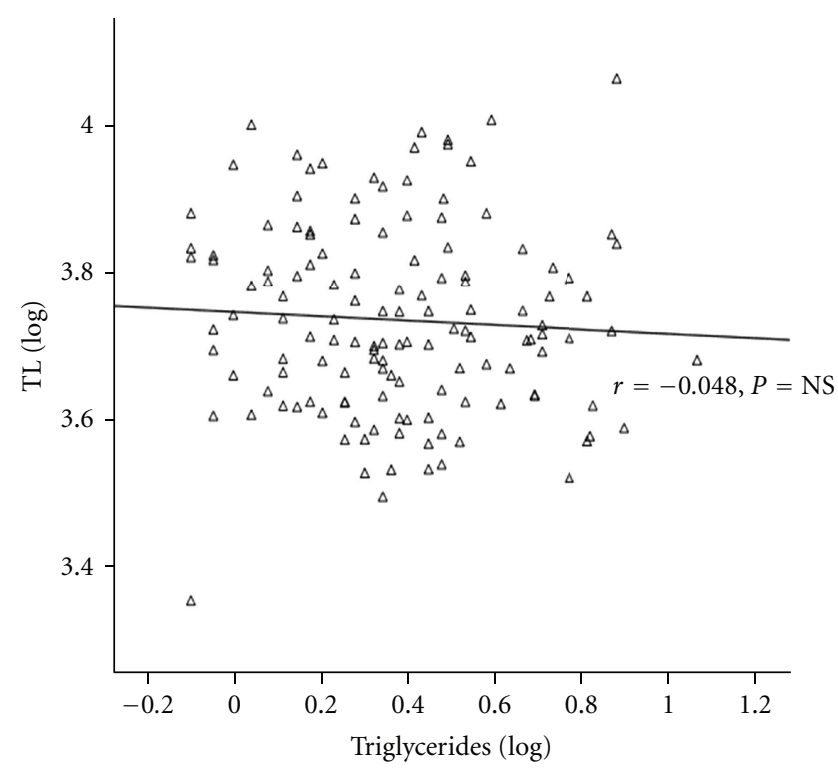

(a)

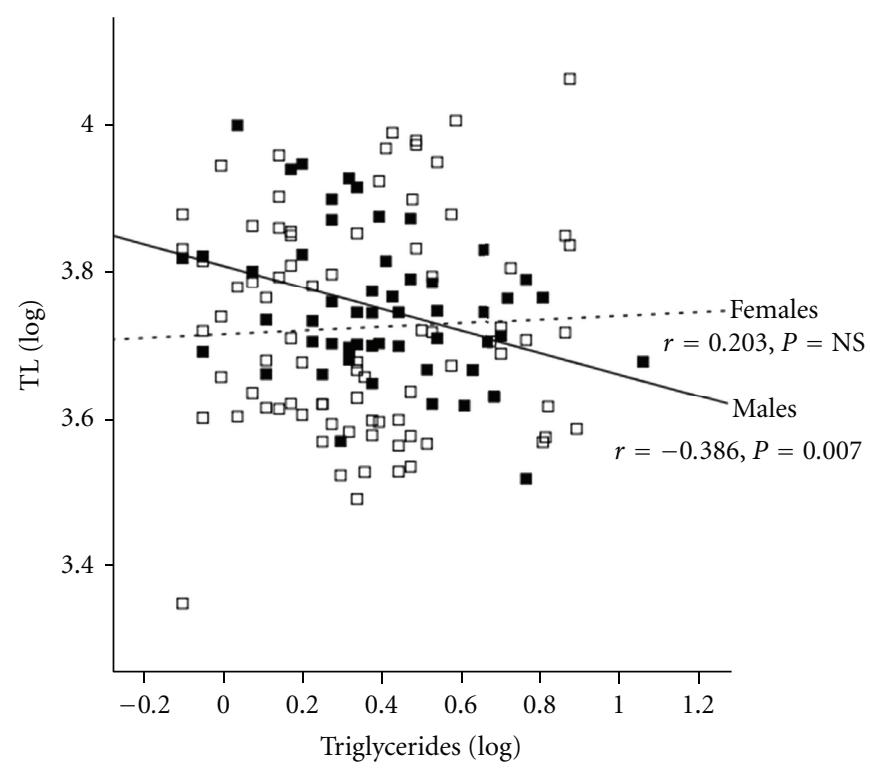

(b)

Figure 3: The relationship between triglycerides and TL in South Asian T2DM group. (a) Linear regression analysis of triglycerides and TL in T2DM (clear triangles); (b) linear regression analysis of triglycerides and TL in T2DM by gender (male: dark squares and solid line, females: open squares and dotted line).

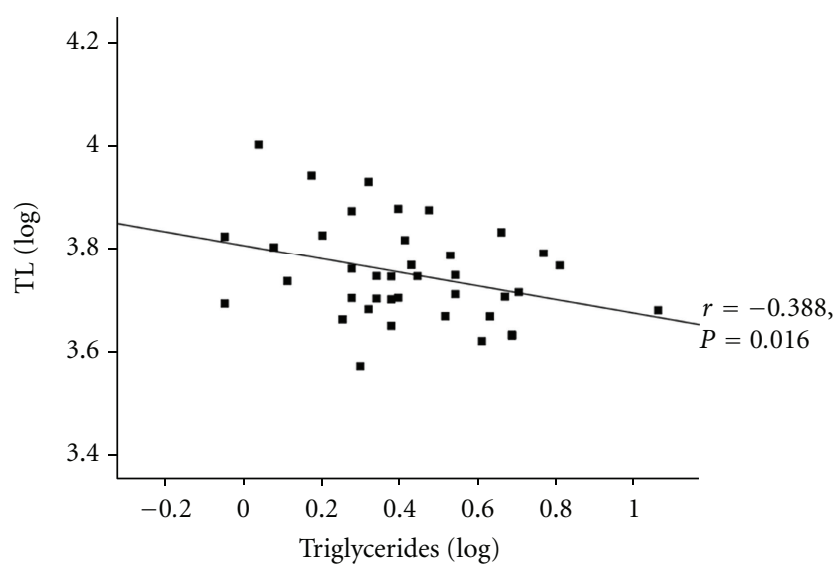

FIGURE 4: TL in obese male T2DM subjects (BMI: $25-34.9 \mathrm{~kg} / \mathrm{m}^{2}$ ) is inversely correlated with triglycerides $(N=39)$.

pathways in the regulation of longevity appears to be endocrine signalling - many mutations that extend lifespan in animal studies involve homologues of insulin and insulinlike growth factor signalling, genes that were observed to extend lifespan by up to 6 -fold in model studies [30, 31].

Several hypotheses explain the gender difference in TL amongst non-T2DM subjects in this study. First is the higher stress exposure for males and the protective effect of oestrogen in females. Males are more vulnerable to acute and chronic stress in animal models, as well as in lower occupational class workers, compared with females who are more sensitive to stress but nevertheless more resilient [32, 33]. The presence of chronic psychological stress in modern-day adults is, in part, related to a fast-paced modern lifestyle, which significantly contributes to obesity and dysmetabolic syndrome-related ageing, as it can lead to overeating. Furthermore, coelevation of cortisol and insulin causes accumulation of visceral fat over time, which harbours a biochemical environment conducive to several ageing mechanisms that lead to cell senescence $[28,34]$. Benetos and colleagues further explained that at any given chronological age, biological ageing is more advanced in men compared to women, since only in men was TL observed to influence pulse pressure, an index of arterial ageing, hence the conclusion that arterial ageing is modified by gender [35]. In addition, oestrogen deficiency has been shown, in animal models, to inhibit telomerase and, ultimately, lead to telomere shortening [36, 37]. Considering that most, if not all, T2DM women involved in this study were postmenopausal, the protective effects of oestrogen are lost and therefore women, at this stage, tend to catch up with men in the expression of age-related diseases, including telomere attrition.

The significant inverse association of TL with several key lipid parameters (total cholesterol and triglycerides) in South Asian men strengthens the premise that biological ageing is heightened in males, as compared with females of the same chronological age [35]. Differences in triglycerides and HDL-cholesterol between South Asian men and women have also been documented [38], with higher CHD risk for South Asian men as compared to African-Caribbean, white Europeans, and African and Hispanic Americans [39, 40]. The increased predisposition of South Asians to age-related chronic diseases is attributed not only to genetic susceptibility, but also to differences in fat deposition, high physical inactivity and differences in diet [40-42]. 
The atherogenic properties of elevated cholesterol and triglycerides confer repeated mechanical, hemodynamic, and/or immunological injury and, as such, may cause augmented cell turnover and increased production of ROS in certain cells [28]. The link between cholesterol and TL may be secondary to increased cell damage and turnover, which in turn amplifies cell ageing by bringing cells to their maximum replicative capacity-translating to shortened TL. This could also tie in with age-related innate immune pathway activation in adipose tissue and its link with subclinical chronic inflammation [15].

This study has several limitations. Causality cannot be inferred from this work due to the cross-sectional nature of the study. Furthermore, while most of our findings are confirmatory, it is noteworthy that South Asians are unique compared to other ethnicities, as several factors that include gender discrepancy and metabolic parameters (such as total cholesterol and triglycerides) appear to play a role in the complex maintenance of TL. These observations were either noted in both genders in other ethnic groups or not at all.

In summary, male South Asians with T2DM have shorter TL when compared with their non-T2DM counterparts, and this correlates with triglycerides and cholesterol levels in the same subgroup. This study highlights enhanced biological ageing among South Asian T2DM men, which appears to be tracked by changes in lipids and BMI. This suggests that raised lipids and BMI could directly contribute to premature ageing; however, this possibility needs further study.

\section{Acknowledgments}

The authors are grateful to UK Asian Diabetes study and WHSS study groups. The authors would also like to thank Birmingham Science City for their support as well as the Warwickshire Institute for the study of Diabetes and Endocrinology. They also thank the College of Science Research Center in King Saud University as well as King Abdul Aziz City for Science and Technology (KACST) in Riyadh, Saudi Arabia, for their support.

\section{References}

[1] A. Misra and L. Khurana, "Obesity-related non-communicable diseases: South Asians vs White Caucasians," International Journal of Obesity, vol. 35, no. 2, pp. 167-187, 2011.

[2] J. W. Lee, F. L. Brancati, and H. C. Yeh, "Trends in the prevalence of type 2 diabetes in Asians versus whites: results from the United States National Health Interview Survey, 19972008," Diabetes Care, vol. 34, no. 2, pp. 353-357, 2011.

[3] S. N. Wulan, K. R. Westerterp, and G. Plasqui, "Ethnic differences in body composition and the associated metabolic profile: a comparative study between Asians and Caucasians," Maturitas, vol. 65, no. 4, pp. 315-319, 2010.

[4] L. Zhang, Q. Qiao, J. Tuomilehto et al., "Distinct ethnic differences in lipid profiles across glucose categories," Journal of Clinical Endocrinology and Metabolism, vol. 95, no. 4, pp. 1793-1801, 2010.

[5] S. Bellary, J. O’Hare, N. Raymond et al., "Enhanced diabetes care to patients of south Asian ethnic origin (the United Kingdom Asian Diabetes Study): a cluster randomised con- trolled trial," The Lancet, vol. 371, no. 9626, pp. 1769-1776, 2008.

[6] Z. Songyang, "Introduction to telomeres and telomerase," Methods in Molecular Biology, vol. 735, pp. 1-11, 2011.

[7] P. Martínez and M. A. Blasco, "Telomeric and extra-telomeric roles for telomerase and the telomere-binding proteins," Nature Reviews Cancer, vol. 11, no. 3, pp. 161-176, 2011.

[8] D. T. Eisenberg, "An evolutionary review of human telomere biology: the thrifty telomere hypothesis and notes on potential adaptive paternal effects," American Journal of Human Biology, vol. 23, no. 2, pp. 149-167, 2011.

[9] R. M. Marion and M. A. Blasco, "Telomeres and telomerase in adult stem cells and pluripotent embryonic stem cells," Advances in Experimental Medicine and Biology, vol. 695, pp. 118$131,2010$.

[10] A. V. Diez Roux, N. Ranjit, N. S. Jenny et al., "Race/ethnicity and telomere length in the Multi-Ethnic Study of Atherosclerosis," Aging Cell, vol. 8, no. 3, pp. 251-257, 2009.

[11] A. Falus, I. Marton, E. Borbenyi et al., "The 2009 Noble Prize in Medicine and its surprising message: lifestyle is associated with telomerase activity," Orvosi Hetilap, vol. 151, no. 24, pp. 965-970, 2010.

[12] A. G. Mainous 3rd, C. J. Everett, V. A. Diaz et al., "Leukocyte telomere length and marital status among middle-aged adults," Age and Ageing, vol. 40, no. 1, pp. 73-78, 2011.

[13] Y. Y. Wang, A. F. Chen, H. Z. Wang, L. Y. Xie, K. X. Sui, and Q. -Y. Zhang, "Association of shorter mean telomere length with large artery stiffness in patients with coronary heart disease," Aging Male, vol. 14, no. 1, pp. 27-32, 2011.

[14] K. D. Salpea and S. E. Humphries, "Telomere length in atherosclerosis and diabetes," Atherosclerosis, vol. 209, no. 1, pp. 3538, 2010.

[15] O. S. Al-Attas, N. M. Al-Daghri, M. S. Alokail et al., "Adiposity and insulin resistance correlate with telomere length in middle-aged Arabs: the influence of circulating adiponectin," European Journal of Endocrinology, vol. 163, no. 4, pp. 601-607, 2010.

[16] I. M. Wentzensen, L. Mirabello, R. M. Pfeiffer, and S. A. Savage, "The association of telomere length and cancer: a metaanalysis," Cancer Epidemiology Biomarkers and Prevention, vol. 20, no. 6, pp. 1238-1250, 2011.

[17] R. Y. L. Zee, A. J. Castonguay, N. S. Barton, S. Germer, and M. Martin, "Mean leukocyte telomere length shortening and type 2 diabetes mellitus: a case-control study," Translational Research, vol. 155, no. 4, pp. 166-169, 2010.

[18] A. H. Barnett, A. N. Dixon, S. Bellary et al., "Type 2 diabetes and cardiovascular risk in the UK south Asian community," Diabetologia, vol. 49, no. 10, pp. 2234-2246, 2006.

[19] J. P. O'Hare, N. T. Raymond, S. Mughal et al., "Evaluation of delivery of enhanced diabetes care to patients of South Asian ethnicity: the United Kingdom Asian Diabetes Study (UKADS)," Diabetic Medicine, vol. 21, no. 12, pp. 1357-1365, 2004.

[20] F. P. Cappuccio, D. G. Cook, R. W. Atkinson, and P. Strazzullo, "Prevalence, detection, and management of cardiovascular risk factors in different ethnic groups in South London," Heart, vol. 78, no. 6, pp. 555-563, 1997.

[21] M. A. Miller, S. M. Kerry, Y. Dong, G. A. Sagnella, D. G. Cook, and F. P. Cappuccio, "Circulating soluble E-selectin levels and the Ser128Arg polymorphism in individuals from different ethnic groups," Nutrition, Metabolism and Cardiovascular Diseases, vol. 15, no. 1, pp. 65-70, 2005.

[22] R. M. Cawthon, "Telomere measurement by quantitative PCR," Nucleic Acids Research, vol. 30, no. 10, p. e47, 2002. 
[23] Z. Yang, X. Huang, H. Jiang et al., "Short telomeres and prognosis of hypertension in a Chinese population," Hypertension, vol. 53, no. 4, pp. 639-645, 2009.

[24] X. Liu, G. Bao, T. Huo, Z. Wang, X. He, and G. Dong, "Constitutive telomere length and gastric cancer risk: casecontrol analysis in Chinese Han population," Cancer Science, vol. 100, no. 7, pp. 1300-1305, 2009.

[25] K. Nordfjäll, U. Svenson, K. F. Norrback, R. Adolfsson, P. Lenner, and G. Roos, "The individual blood cell telomere attrition rate is telomere length dependent," PLoS Genetics, vol. 5, no. 2, Article ID e1000375, 2009.

[26] J. Z. Guan, T. Maeda, M. Sugano, J. I. Oyama, Y. Higuchi, and N. Makino, "Change in the telomere length distribution with age in the Japanese population," Molecular and Cellular Biochemistry, vol. 304, no. 1-2, pp. 353-360, 2007.

[27] O. S. Al-Attas, N. Al-Daghri, A. Bamakhramah, S. Shaun Sabico, P. McTernan, and T. T. K. Huang, "Telomere length in relation to insulin resistance, inflammation and obesity among Arab youth," Acta Paediatrica, vol. 99, no. 6, pp. 896899, 2010.

[28] E. S. Epel, "Psychological and metabolic stress: a recipe for accelerated cellular aging?" Hormones, vol. 8, no. 1, pp. 7-22, 2009.

[29] A. Avogaro, S. V. De Kreutzenberg, and G. P. Fadini, "Insulin signaling and life span," Pflugers Archiv, vol. 459, no. 2, pp. 301-314, 2010.

[30] T. Minamino and I. Komuro, "Hyperinsulinemia and aging," Nippon Rinsho, vol. 65, no. 6, pp. 1147-1154, 2007.

[31] W. S. Browner, A. J. Kahn, E. Ziv et al., "The genetics of human longevity," American Journal of Medicine, vol. 117, no. 11, pp. 851-860, 2004.

[32] H. Cohen and R. Yehuda, "Gender differences in animal models of posttraumatic stress disorder," Disease Markers, vol. 30, no. 2-3, pp. 141-150, 2011.

[33] A. Tsutsumi, K. Kayaba, and S. Ishikawa, "Impact of occupational stress on stroke across occupational classes and genders," Social Science and Medicine, vol. 72, no. 10, pp. 1652 1658, 2011.

[34] G. P. Chrousos, "The role of stress and the hypothalamicpituitary-adrenal axis in the pathogenesis of the metabolic syndrome: neuro-endocrine and target tissue-related causes," International Journal of Obesity, vol. 24, no. 2, pp. S50-S55, 2000.

[35] A. Benetos, K. Okuda, M. Lajemi et al., "Telomere length as an indicator of biological aging the gender effect and relation with pulse pressure and pulse wave velocity," Hypertension, vol. 37, no. 2, pp. 381-385, 2001.

[36] J. Lin, C. H. Kroenke, E. Epel et al., "Greater endogenous estrogen exposure is associated with longer telomeres in postmenopausal women at risk for cognitive decline," Brain Research, vol. 1379, pp. 224-231, 2011.

[37] S. Bayne, M. E. E. Jones, H. Li, A. R. Pinto, E. R. Simpson, and J. P. Liu, "Estrogen deficiency leads to telomerase inhibition, telomere shortening and reduced cell proliferation in the adrenal gland of mice," Cell Research, vol. 18, no. 11, pp. 11411150, 2008 .

[38] R. Kolluri, D. Pinedo, A. Edmondson-Holt, K. S. Grewal, and J. M. Falko, "Dyslipidemia in South Asians living in a western community," Journal of Clinical Lipidology, vol. 3, no. 1, pp. 14-18, 2009.

[39] T. Tillin, N. Forouhi, D. G. Johnston, P. M. McKeigue, N. Chaturvedi, and I. F. Godsland, "Metabolic syndrome and coronary heart disease in South Asians, African-Caribbeans and white Europeans: a UK population-based cross-sectional study," Diabetologia, vol. 48, no. 4, pp. 649-656, 2005.

[40] K. C. Ferdinand, "Managing cardiovascular risk in minority patients," Journal of the National Medical Association, vol. 97, no. 4, pp. 459-466, 2005.

[41] S. Bellary, J. Paul O'Hare, N. T. Raymond et al., "Premature cardiovascular events and mortality in south Asians with type 2 diabetes in the United Kingdom Asian Diabetes Study effect of ethnicity on risk," Current Medical Research and Opinion, vol. 26, no. 8, pp. 1873-1879, 2010.

[42] M. Balasubramanyam, A. Adaikalakoteswari, S. F. Monickaraj, and V. Mohan, "Telomere shortening and metabolic/vascular diseases," Indian Journal of Medical Research, vol. 125, no. 3, pp. 441-450, 2007. 


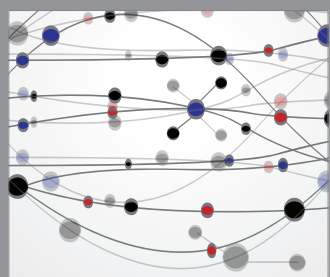

The Scientific World Journal
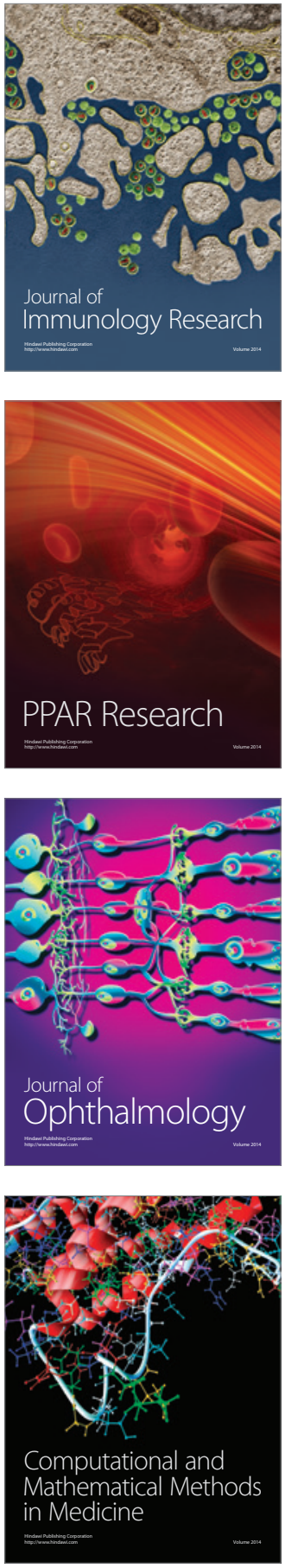

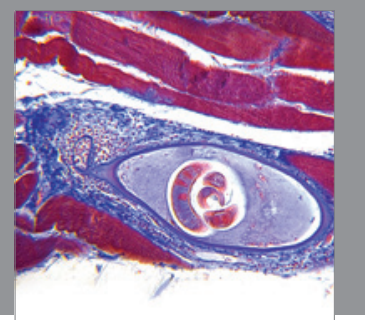

Gastroenterology

Research and Practice
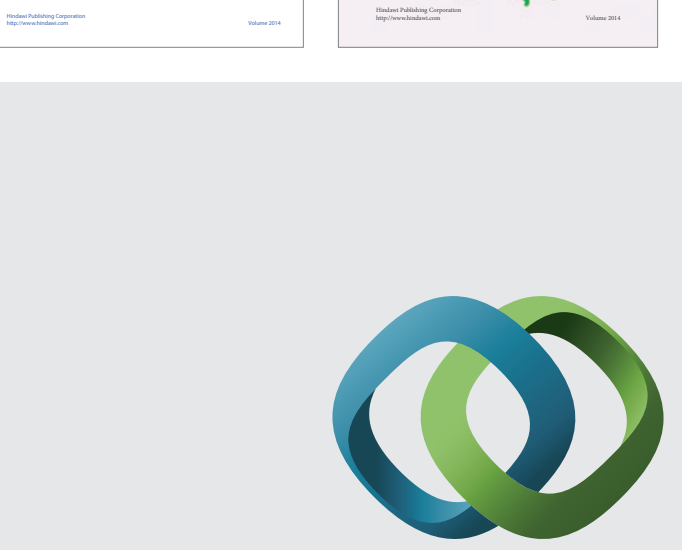

\section{Hindawi}

Submit your manuscripts at

http://www.hindawi.com
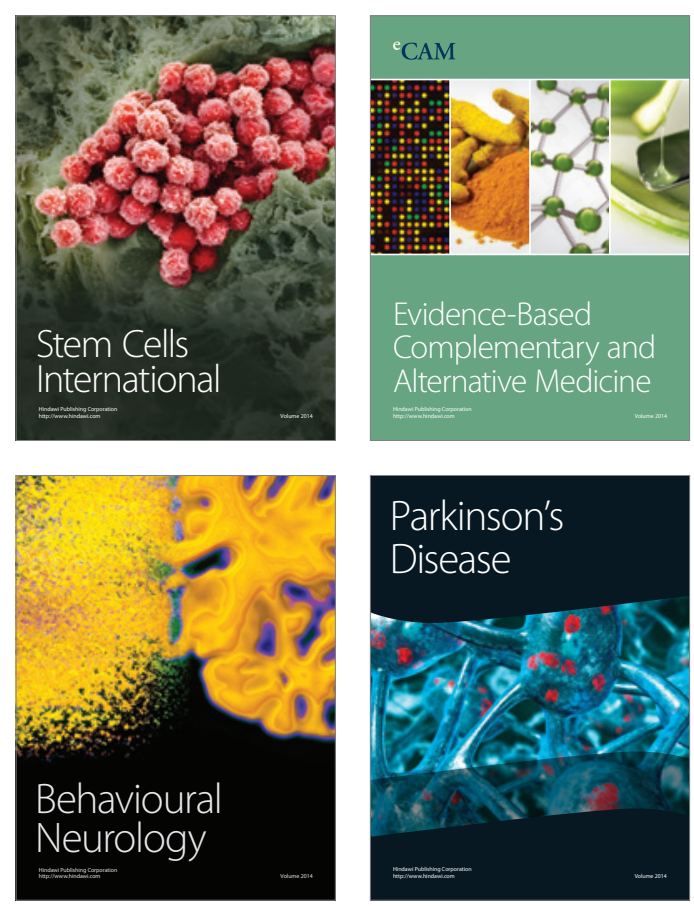

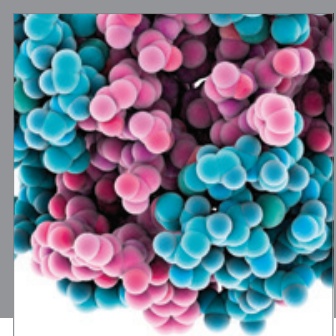

Journal of
Diabetes Research

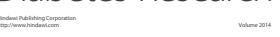

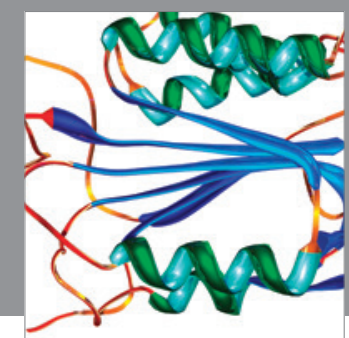

Disease Markers
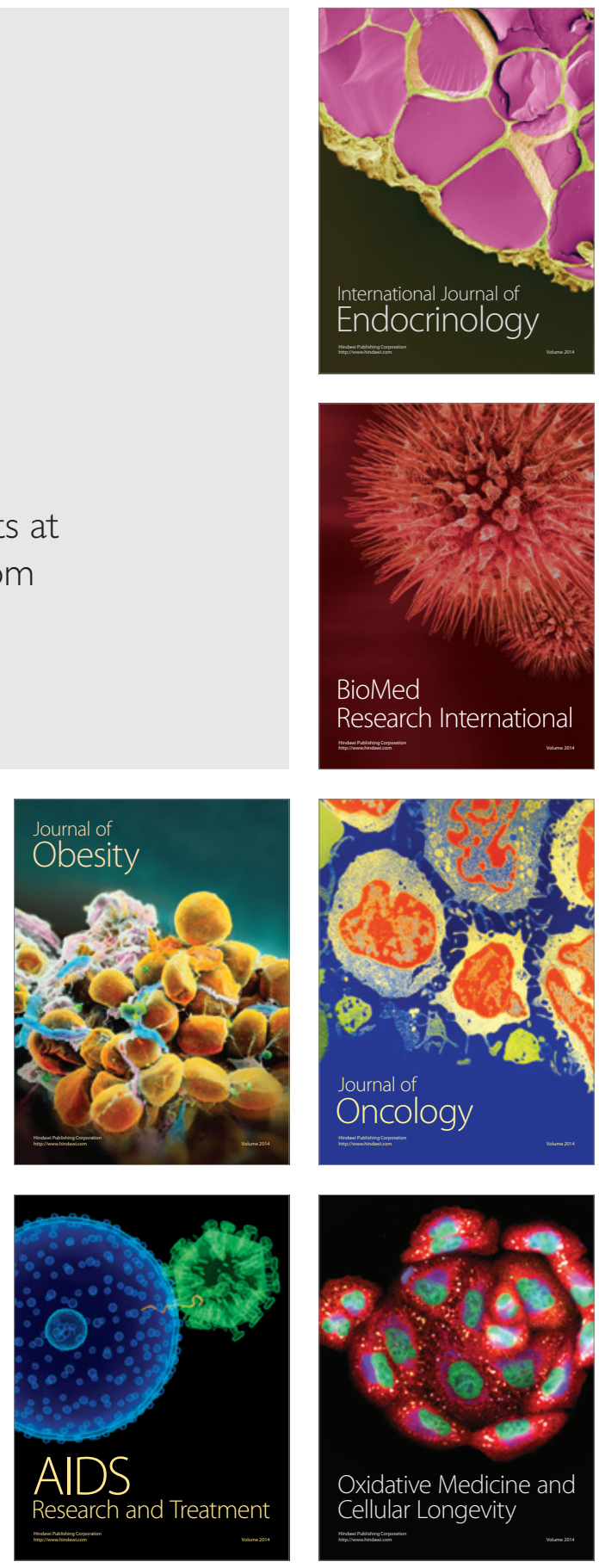